\title{
1 Cool-SPS stabilization and sintering of thermally fragile, potentially
}

2 magnetoelectric, $\mathrm{NH}_{4} \mathrm{FeP}_{2} \mathrm{O}_{7}(C J-3: I L 04)$

3 T. Herisson de Beauvoir, A. Sangregorio, A. Bertrand, C. Payen, D. Michau, U-C. Chung, M. Josse*

\section{Abstract}

5 Cool-SPS conditions (low temperature/high pressure) can be used to densify materials with limited 6 thermodynamical stability, due to low temperature phase transition, melting, decomposition... We 7 recently demonstrated the potential of SPS for the stabilization and densification of diversified materials 8 at very low temperatures. Here we report the sintering of a potentially magnetoelectric pyrophosphate 9 with low temperature decomposition. Characterization of its magnetic properties are presented, and 10 dielectric/magnetoelectric characterization could also be performed thanks to the high densification 11 obtained at temperatures as low as $200{ }^{\circ} \mathrm{C}$.

\section{Introduction}

13 Low temperature ceramic sintering techniques have recently been developed and showed extraordinary 14 potential for accessing high density at extremely low temperatures, typically below $400{ }^{\circ} \mathrm{C}$, including 15 Cold Sintering Process [1-7], Hydrothermal Hot Pressing [8-10] and Cool-SPS [11,12]. Beyond the 16 energy and cost reduction offered by temperature reduction [13], multiple gaps are bridged by such 17 processes. Indeed, such low processing temperatures open new possibilities such as the formation of 18 novel polymer/ceramic composite materials, with ceramic as a major phase [14-16]. This also enables the 19 formation of novel LTCC materials [17]. Other perspectives have recently been recently explored in the 20 case of Cool-SPS, highlighting additional advantages of these low temperature processing techniques. 21 One of them lies in the applied pressure necessary to realize such processes. In prior works, we observed 22 that materials stability ranges may be modified under applied mechanical pressure, enabling stabilization 23 of thermodynamically fragile phases - such as compounds facing low temperature decomposition, 24 melting or irreversible phase transition [12] - and their efficient sintering (by Cool-SPS) resulting in very 25 high density (typically above $95 \%$ of theoretical density). Another one is the opportunity to use, and also 26 sinter efficiently, hydrated precursors [12], by controlling the dehydration through optimized sintering 27 cycles [11]. One may also be able to perform in situ synthesis of phases that cannot be synthesized 28 quantitatively otherwise [12]. Such potentialities create an opportunity to diversify the chemicals classes 29 of materials investigated for diverse properties (physical, chemical...). Exploring these low temperature 30 processing techniques can also allow for breakthroughs such as the recent elaboration of the first 31 molecular ceramic [18]. One can thus foresee the potential of low temperature sintering methods for 
bridging processing gaps (elaboration at low temperature), chemical gaps (enabling investigation of more diversified families of compounds), or even shaping gaps (processing of molecular ceramics previously unknown).

Indeed, we are now able to obtain dense materials from powders that were up to now considered to be impossible to sinter, and thus characterized as powder, films or crystals, with the inherent processing demands and characterization limitations of these shapings ${ }^{1}$. Widening the span of sinterable materials opens up interesting opportunities, especially in sulfate and phosphate compounds. They usually show low temperature stability, making their processing more difficult than refractory oxides. This concerns various applications such as electronic properties for batteries [19] or even mechanical ones in the process of biomaterials [20]. Using Cool-SPS among these low temperature processing techniques, it was possible to highlight magnetoelectric properties of $\mathrm{MnSO}_{4}$ ceramics that could not be obtained through conventional sintering [11]. When looking for magnetoelectric properties in single phase materials, it becomes interesting to use low temperature sintering techniques to densify materials which crystalchemical and magnetic properties allow for a potential magnetoelectric coupling. In the present study, we focus our interest on $\mathrm{NH}_{4} \mathrm{FeP}_{2} \mathrm{O}_{7}$, once reported for its structure [21]. It completes the series of iron pyrophosphates $\mathrm{AFeP}_{2} \mathrm{O}_{7}(\mathrm{~A}=\mathrm{Li}, \mathrm{Na}, \mathrm{K}, \mathrm{Rb}, \mathrm{Cs}, \mathrm{Ag})$ [22-25], which display antiferromagnetic phase transitions below room temperature thanks to super-super exchange interactions [22]. $\mathrm{NH}_{4} \mathrm{FeP}_{2} \mathrm{O}_{7}$ is also isostructural to $\mathrm{KCrP}_{2} \mathrm{O}_{7}$, a pyrophosphate which symmetry allows for a magnetoelectric coupling [26], and this is an indictment to explore similar properties in the title compound. The main challenge to test such a magnetoelectric coupling is to obtain a highly densified ceramic to allow for dielectric characterization and avoid spurious contributions related to poor densification. To reach this objective, we propose to use Cool-SPS an efficient technique to obtain both high density and phase stabilization [12].

\section{Experimental section}

$\mathrm{NH}_{4} \mathrm{FeP}_{2} \mathrm{O}_{7}$ synthesis is performed through solid state reaction of a green pellet constituted of both $\left(\mathrm{NH}_{4}\right)_{2} \mathrm{HPO}_{4}$ (Sigma Aldrich 98\%) and $\alpha-\mathrm{Fe}_{2} \mathrm{O}_{3}$. The latter is obtained from the heat treatment of $\mathrm{Fe}\left(\mathrm{NO}_{3}\right)_{3} \cdot 9 \mathrm{H}_{2} \mathrm{O}$ (Sigma Aldrich $98 \%$ ) at $500{ }^{\circ} \mathrm{C}$ overnight, as commercial $\alpha-\mathrm{Fe}_{2} \mathrm{O}_{3}$ presents a poor reactivity and its use results in an important amount of non-reacted $\alpha-\mathrm{Fe}_{2} \mathrm{O}_{3}$. The two powders are mixed in an agate mortar and pelletized, then they are fired in a furnace at $210^{\circ} \mathrm{C}$ for $24 \mathrm{~h}$. Sealed nitrogen

\footnotetext{
${ }^{1}$ The processing of films requires compatible methods and substrates, the growth of single crystals require specific methods and time, both can require multiple-step processing. Characterization methods requiring electrical contact, dense samples (i.e. dielectric measurements...) are generally impossible for powders. The characterization of films frequently require specific methods for data collection, analysis and interpretation. The characterization of crystal can be limited by their inherent anisotropy (except for the cubic crystal system) and growth habit (acicular growth, platelets...) and may require complex preparation steps (oriented cutting, polishing...).
} 
environment, as well as nitrogen flux, were used to perform the synthesis, but resulted in a high content of remaining iron oxide, and sometimes promoted the formation of other phosphates as secondary phases.

62 Using a sealed primary vacuum atmosphere allowed to obtain the higher ratio of $\mathrm{NH}_{4} \mathrm{FeP}_{2} \mathrm{O}_{7}$ over $\alpha$ $63 \mathrm{Fe}_{2} \mathrm{O}_{3}$. When a stoichiometric ratio of $\left(\mathrm{NH}_{4}\right)_{2} \mathrm{HPO}_{4}$ and iron oxide is used, there is an important part of 64 remaining iron oxide, due to the volatilization of ammonium phosphate. Adding an excess of $\left(\mathrm{NH}_{4}\right)_{2} \mathrm{HPO}_{4}$ 65 allows to compensate this volatilization, up to a point were other phases appear. The best compromise was found to be a $20 \mathrm{wt} . \%$ excess of $\left(\mathrm{NH}_{4}\right)_{2} \mathrm{HPO}_{4}$, although traces of $\alpha-\mathrm{Fe}_{2} \mathrm{O}_{3}$ are still visible on the XRD pattern of the as-synthesized product. After synthesis, the powder was placed in a SPS sintering die made of WC and surrounded by Ta foils to avoid any cross contamination between the die and the powder.

Dense ceramics were prepared with Spark plasma sintering (Dr. Sinter Lab spark plasma sintering system, Model SPS-511S/SPS-515S) in WC:Co $10 \mathrm{~mm}$ die using $0.5 \mathrm{~g}$ of powder for each sintering attempt. Thermocouple is used to monitor temperature, in contact with the inner part of the die. 12 ON/2 OFF pulse sequence is applied for all samples. Densification was continuously monitored by the displacement of a punch rod. A $30^{\circ} \mathrm{C} / \mathrm{min}$ heating ramp was used for all different sintering experiments, while temperature was varied from $200{ }^{\circ} \mathrm{C}$ to $300{ }^{\circ} \mathrm{C}$. Pressure was set to $300 \mathrm{MPa}$ and sintering time to $10 \mathrm{~min}$ for all samples. Sintering attempts were performed under primary vacuum of about $10 \mathrm{~Pa}$. Powder and pellet diffraction were performed with a Phillips X'Pert MPD X-ray diffractometer with the BraggBrentano geometry using $\mathrm{Cu} \mathrm{K} \alpha 1 / \mathrm{K} \alpha 2$ radiation $\left(10^{\circ}<2 \theta<80^{\circ}\right.$ range, step of $\left.0.017^{\circ}\right)$. The ceramics' relative densities were evaluated by geometrical measurements considering a theoretical density of 2.75 $\mathrm{g} / \mathrm{cm}^{3}$ for $\mathrm{NH}_{4} \mathrm{FeP}_{2} \mathrm{O}_{7}$ [21]. The pellets' microstructures were observed on fracture surfaces, with a scanning electron microscope (JEOL 840 SEM). TGA measurements were performed with a Setaram tag2400 apparatus. Magnetic measurements were performed with a MPMS-7-XL (S.Q.U.I.D) magnetometer (Quantum Design) on ceramics. Zero-field cooled and field cooled data were collected with an applied field of $1 \mathrm{kOe}$ and with increasing temperature from $2 \mathrm{~K}$ to $300 \mathrm{~K}$. Silver electrodes were deposited on both surfaces of the pellets and silver wires were subsequently used for sake of electrical connection. Once prepared for dielectric measurements, the ceramics were placed in a Quantum Design Physical Properties Measurement System (PPMS) from which a $9 \mathrm{~T}$ magnetic field can be applied. The dielectric measurements were carried out in the frequency range of $10^{2}-10^{6} \mathrm{kHz}$ (the amplitude of the applied ac electric field being 1V) using an HP4194a impedance bridge, at heating and cooling rates between 0,2 and $1 \mathrm{~K} / \mathrm{min}$. Measurements of capacitance and dielectric losses were first performed by scanning temperature with and without application of a magnetic field of 9T. In a second step, the magnetic field was swept from $-9 \mathrm{~T}$ to $9 \mathrm{~T}$ at $2 \mathrm{~K}$ to evaluate the magnetoelectric behavior of the samples.

\section{Results and discussion}


XRD data confirm the structural model proposed in the literature [21], and points the difficulty to fully

94 separate as-synthesized $\mathrm{NH}_{4} \mathrm{FeP}_{2} \mathrm{O}_{7}$ phase from $\alpha-\mathrm{Fe}_{2} \mathrm{O}_{3}$ (see figure $1 \mathrm{a}$ and $\mathrm{b}$ ). Whatever the temperature 95 conditions tested for synthesis, $\alpha-\mathrm{Fe}_{2} \mathrm{O}_{3}$ traces remain present after synthesis of the powder. At 96 temperatures higher than $210^{\circ} \mathrm{C}$, decomposition of the target pyrophosphate occurs, and other phosphates 97 are formed. On the opposite, synthesis performed below $210^{\circ} \mathrm{C}$ show a limited reactivity, leading to an 98 important amount of remaining $\alpha-\mathrm{Fe}_{2} \mathrm{O}_{3}$. Therefore, synthesis temperature was set to $210{ }^{\circ} \mathrm{C}$, and $220{ }^{\circ} \mathrm{C}$ 99 is considered as decomposition temperature under vacuum, and is reported on figure $1 \mathrm{c}$ ) as a dashed red 100 line. Despite the presence of hematite in the powder, XRD profile refinements (see table 1) confirm that 101 lattice parameters of $\mathrm{NH}_{4} \mathrm{FeP}_{2} \mathrm{O}_{7}$ phase are consistent with literature data [21]. After the powder was 102 synthesized, it was placed in a WC die to be sintered in Cool-SPS conditions. Sintering temperature 103 ranging from 200 to $300{ }^{\circ} \mathrm{C}$ were used to prepare ceramics. Interestingly, it is observed that the 104 contribution of $\alpha-\mathrm{Fe}_{2} \mathrm{O}_{3}$ traces to XRD patterns is apparently suppressed, as its very presence after 105 sintering can be doubted for ceramics prepared at $250^{\circ} \mathrm{C}$ and above (figure $1 \mathrm{a}$ and $\mathrm{b}$ ). An hypothesis for 106 that observation deals with the presence of $20 \mathrm{wt} . \%$ of ammonium hydrogen phosphate during synthesis, 107 which may remain unreacted, possibly in amorphous form. We know from XRD on as-synthesized 108 powder that $\alpha-\mathrm{Fe}_{2} \mathrm{O}_{3}$ remains in excess. Therefore, Cool-SPS treatment on the as-synthesized powder, in 109 which some precursors remain, likely promotes their reactivity while sintering is performed, ultimately 110 resulting in the formation of pure, crystalized $\mathrm{NH}_{4} \mathrm{FeP}_{2} \mathrm{O}_{7}$ when Cool-SPS is performed at $300^{\circ} \mathrm{C}$. This 111 makes the present processing route a reactive sintering, which probably contributes to the high density 112 obtained, despite the very low temperature used, down to $200{ }^{\circ} \mathrm{C}$, even below the synthesis temperature. 113 From table 1, we can confirm the preservation of $\mathrm{NH}_{4} \mathrm{FeP}_{2} \mathrm{O}_{7}$ lattice parameters, thus phase purity. Table 1141 also displays densities of pellets sintered at temperatures ranging from 200 to $300{ }^{\circ} \mathrm{C}$. All of them show 115 a density above $94 \%$, even reaching $98 \%$ for a $300{ }^{\circ} \mathrm{C}$ sintering treatment. Thanks to Cool-SPS 116 efficiency, short sintering time (10 min only) and very low sintering temperatures are sufficient to obtain 117 highly dense ceramic materials. These observations confirm the possibility of using SPS in such low 118 temperature conditions, to extend the stability range of some compound, and to rely on reactive sintering 119 in Cool-SPS conditions.

120 Moreover, considering thermal analysis performed on the initial powder (figure 1c), decomposition in air 121 occurs in three steps ranging from 170 to $600{ }^{\circ} \mathrm{C}$. The important information to consider in the present 122 case it the starting decomposition temperature, $170{ }^{\circ} \mathrm{C}$. This corresponds to $130{ }^{\circ} \mathrm{C}$ below the sintering 123 temperature. However, we know from investigating its synthesis conditions that $\mathrm{NH}_{4} \mathrm{FeP}_{2} \mathrm{O}_{7}$ is more 124 stable under vacuum than in air. Decomposition temperature has previously been determined as $220^{\circ} \mathrm{C}$ 125 under vacuum. Considering now the highest sintering temperature used to obtain a $98 \%$ dense sample, 
this represents $80{ }^{\circ} \mathrm{C}$ above the decomposition temperature under vacuum. It suggests a modified thermal

127 stability range in Cool-SPS conditions compared to atmospheric pressure conditions, which has already 128 been pointed out for other materials [12]. As the refined lattice parameters of the ceramics (table 1), 129 obtained from profile refinements (figure 1 a), show very good consistency with the literature and from 130 one sample to the other, it is clear that Cool-SPS processing above decomposition temperature preserves 131 (and indeed increase) the phase purity from as-synthesized powder to $300{ }^{\circ} \mathrm{C}$ sintered ceramic of $132 \mathrm{NH}_{4} \mathrm{FeP}_{2} \mathrm{O}_{7}$. This confirms the preservation of the phase $80{ }^{\circ} \mathrm{C}$ above its decomposition temperature in 133 vacuum and allows using the obtained samples for further physical characterizations.

134 SEM images were taken on fractured pellets to observe their microstructure. According to these observation (figure 2), no open porosity is observed, and the high densifications geometrically measured are confirmed. Considering the quality of the pure and dense ceramics prepared, physical characterization

137 can be performed on the sintered samples. In the following, all characterizations presented correspond to $138300{ }^{\circ} \mathrm{C}$ sintered pellet, which is both the most dense and most pure specimen at our disposal. Since no 139 magnetic characterization of $\mathrm{NH}_{4} \mathrm{FeP}_{2} \mathrm{O}_{7}$ has been reported previously, the present work is an opportunity 140 to complete the data concerning the $\mathrm{AFeP}_{2} \mathrm{O}_{7}$ series $(\mathrm{A}=\mathrm{Li}, \mathrm{Na}, \mathrm{K}, \mathrm{Rb}, \mathrm{Cs}, \mathrm{Ag})$. Figure 3 displays zero 141 field cool magnetic measurement revealing a maximum of magnetization of $0.17 \mathrm{emu}^{-1}$ at $21 \mathrm{~K}$, and 142 thus a transition to an antiferromagnetic phase below that point. The Curie-Weiss fit perfectly describes 143 the sample magnetic behaviour at high temperature in the paramagnetic state, with $\mathrm{C}=1.19 \mathrm{emu} \cdot \mathrm{K} \cdot \mathrm{mol}^{-1}$ 144 and $\theta=-32.9 \mathrm{~K}$, confirming the antiferromagnetic nature of magnetic interactions in $\mathrm{NH}_{4} \mathrm{FeP}_{2} \mathrm{O}_{7}$. These 145 observations are consistent with literature data on $\mathrm{AFeP}_{2} \mathrm{O}_{7}(\mathrm{~A}=\mathrm{Li}, \mathrm{Na}, \mathrm{K}, \mathrm{Cs}, \mathrm{Ag})$ [22-25], which are 146 recalled in table 2 along with the contribution from this work. Most representatives of this series show 147 antiferromagnetic transitions at low temperatures, and all of them present negative value of $\theta$, indicative 148 of antiferromagnetic interactions. Our data places the ammonium representative as the one displaying the 149 weakest magnetic interactions in the series from this viewpoint, and also show that the magnetic 150 behaviour in this series can not be rationalized on the basis of a simple crystal-chemical parameter such as 151 ionic radius. Since high density pellets can be obtained after sintering, it is now possible to perform 152 reliable dielectric measurements. Figure 4a presents the dielectric measurements performed on pellet 153 sintered at $300{ }^{\circ} \mathrm{C}$, which were found reproducible from several ceramics prepared in the same conditions. 154 According to the small value of the permittivity and the low level of losses (below 0.01), we can tell that 155 the quality of grain boundaries is very good, as defect-related contributions from grain boundaries would 156 otherwise clearly dominate the dielectric response of the sample. A relaxation is observed in the losses 157 from 150 to $100 \mathrm{~K}$, which is associated with a slight decrease in the real part of permittivity. However, the 158 mechanism associated with this dielectric anomaly has not been identified yet, and the small amplitude of 159 permittivity evolution prevents deeper analyses to identify it. As $\mathrm{NH}_{4} \mathrm{FeP}_{2} \mathrm{O}_{7}$ crystal symmetry allows for 
160 a magnetoelectric coupling [26] it is worth trying to investigate it through magnetic field dependent

161 dielectric measurements. On figure $4 \mathrm{~b}$ is displayed the dependence of dielectric permittivity to applied

162 magnetic field, at $300 \mathrm{kHz}$ and $2 \mathrm{~K}$ for the same sample. As can be observed from the plot, a dependence

163 is measured, mostly between \pm 3 to $\pm 6 \mathrm{~T}$, which remains of rather small amplitude, preventing us from

164 performing additional characterization (such as magnetic field dependent polarization) and concluding on

165 its nature. Anyway, this points out the possible presence of a magnetoelectric in the phase, which requires

166 further investigations to be confirmed.

167 Conclusion

168 It was possible to synthesize $\mathrm{NH}_{4} \mathrm{FeP}_{2} \mathrm{O}_{7}$ powder from solid state route, by firing diammonium hydrogen 169 phosphate and iron oxide at $210^{\circ} \mathrm{C}$ under primary vacuum. However hematite $\alpha-\mathrm{Fe}_{2} \mathrm{O}_{3}$ remains present 170 after synthesis, while XRD confirms the stabilization of the target phase, with lattice parameters similar to 171 those reported in the literature [21]. Among the key features of the present work is the behavior of this 172 material during its processing by Cool-SPS. Although no literature data deal with the densification of this 173 material, it was possible to obtain a highly dense material using temperature as low as $200{ }^{\circ} \mathrm{C}$ and 174 sintering time as short as $10 \mathrm{~min}$. This illustrates the potential of low temperature sintering techniques, 175 such as Cool-SPS, for the elaboration of highly densified ceramics. As $\mathrm{NH}_{4} \mathrm{FeP}_{2} \mathrm{O}_{7}$ decomposes in air 176 around $170{ }^{\circ} \mathrm{C}$ and in vacuum around $220^{\circ} \mathrm{C}$, this also illustrates the possibility, pointed previously [12], 177 to densify some thermodynamically fragile materials beyond their thermal stability range, and expand the 178 range of possible characterizations to that allowed by ceramics, which are otherwise mostly limited to 179 studies performed on powder or single-crystals. Moreover, in the present case, although the targeted 180 phase could not be obtained as a pure powder after synthesis, Cool-SPS processing essentially removed 181 the $\alpha-\mathrm{Fe}_{2} \mathrm{O}_{3}$ excess, most probably thanks to reactive sintering. Finally, obtaining such phase-pure, high182 density pellets allowed to perform physical characterizations. Magnetic measurements revealed an 183 antiferromagnetic phase transition at $21 \mathrm{~K}$, consistent with literature data on other members of $\mathrm{AFeP}_{2} \mathrm{O}_{7}$ $184(\mathrm{~A}=\mathrm{Li}, \mathrm{Na}, \mathrm{K}, \mathrm{Cs}, \mathrm{Ag})$ family showing similar properties. Dielectric properties could be measured on 185 dense pellet, confirming the quality of grain boundaries and exhibiting a dielectric anomaly in the range 186 100-150 K. In parallel, magnetic dependence of permittivity exhibits a clue for magnetoelectric coupling 187 in $\mathrm{NH}_{4} \mathrm{FeP}_{2} \mathrm{O}_{7}$, which needs to be further investigated. 

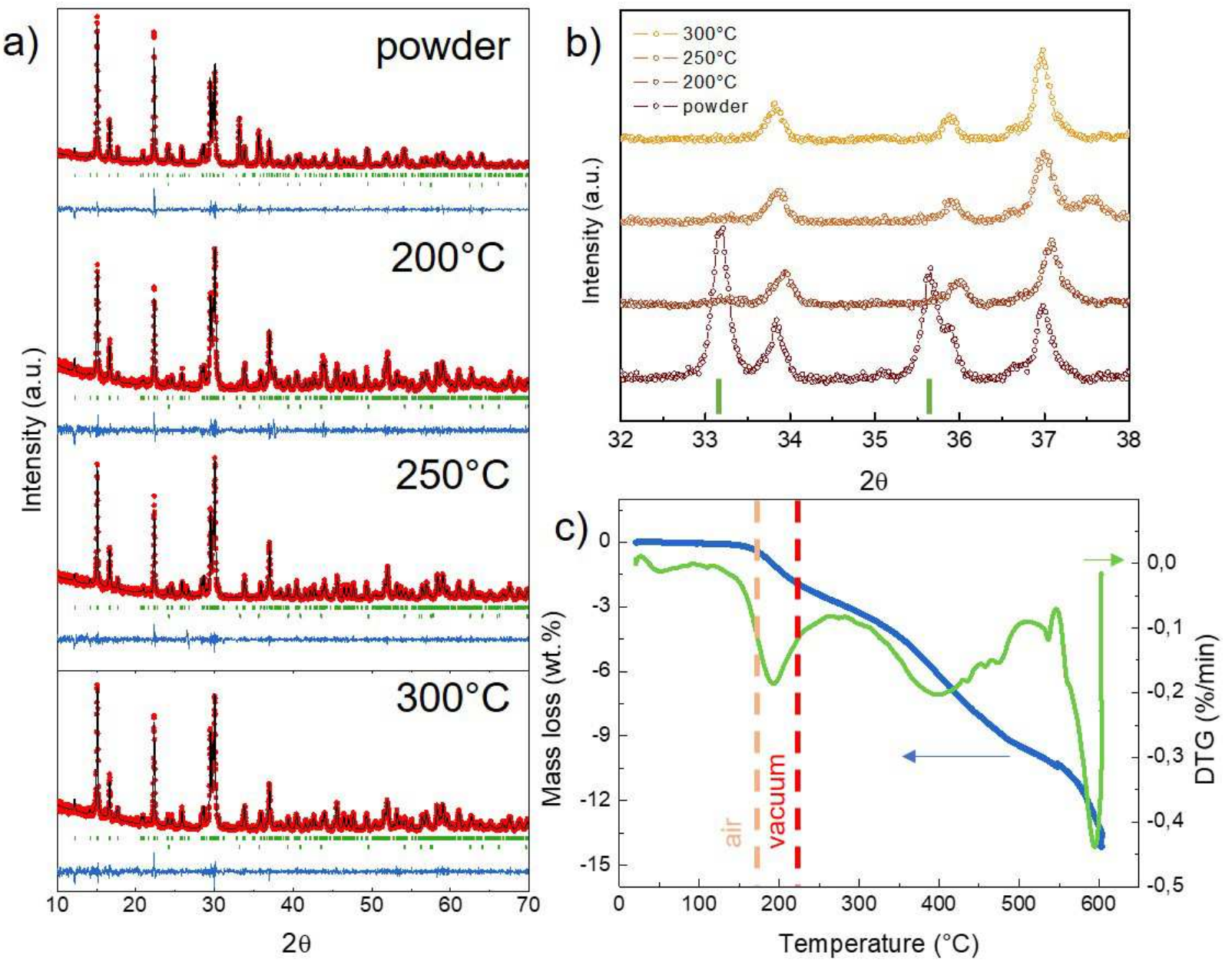

Figure 1: a) XRD patterns collected on synthetized powder and sintered pellets at various temperatures; $b$ ) zoom in the region of $32^{\circ} \leq 2 \theta \leq 38$, with a green mark representing Bragg positions for $\mathrm{Fe}_{2} \mathrm{O}_{3}$ hematite phase (other contributions are due to the title compound) ; c) TGA in air at constant heating rate of $5{ }^{\circ} \mathrm{C} /$ min from room temperature to $600{ }^{\circ} \mathrm{C}$ on $\mathrm{NH}_{4} \mathrm{FeP} \mathrm{P}_{2} \mathrm{O}_{7}$ powder after synthesis (blue line correspond to TG data and green line to $d T G$ ), dashed orange line represents the starting decomposition temperature in air extracted from $T G A\left(170{ }^{\circ} \mathrm{C}\right)$ while red dashed line represents the stability temperature observed in primary vacuum, obtained from synthesis $\left(220^{\circ} \mathrm{C}\right)$ 

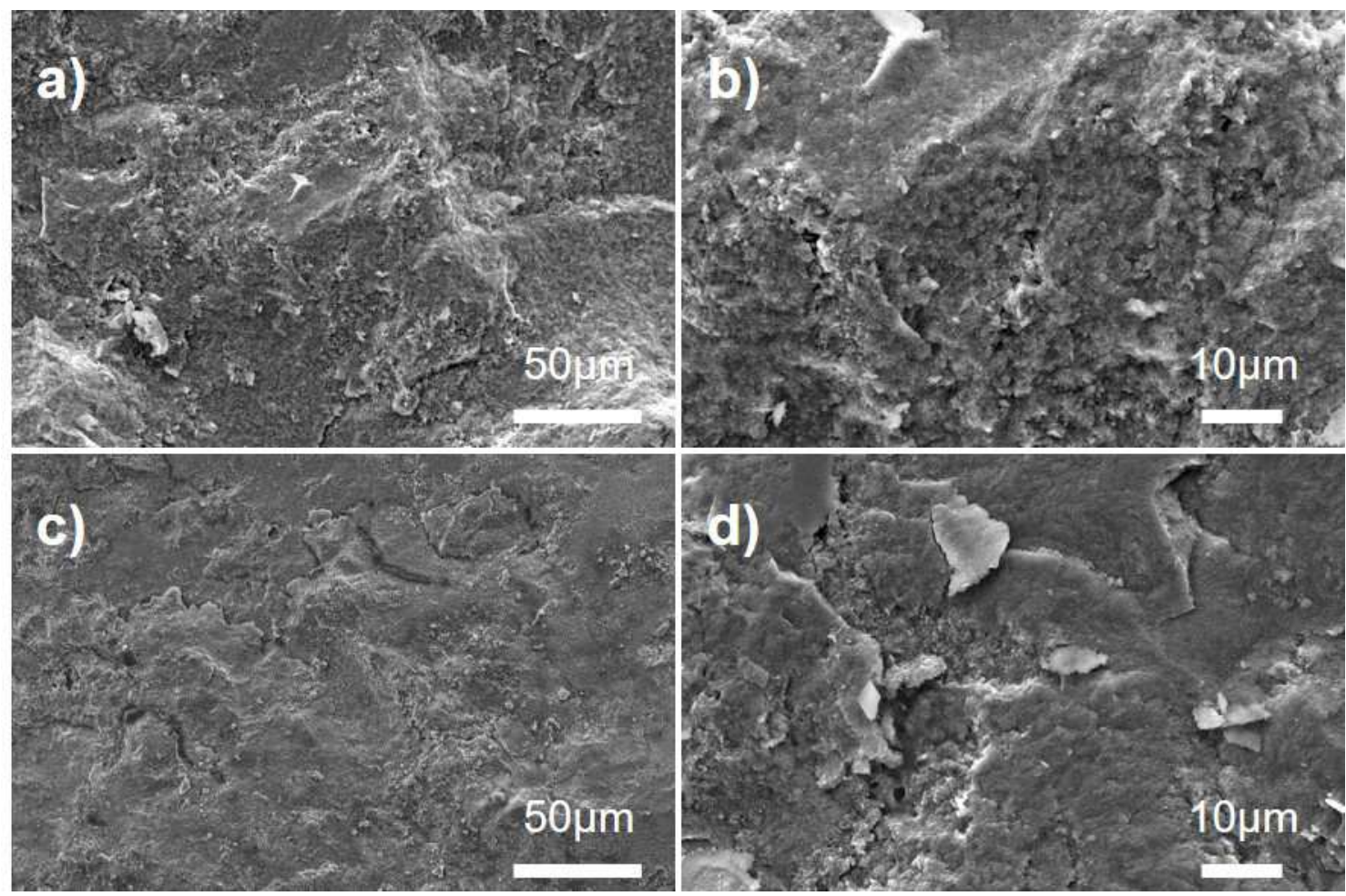

198

Figure 2: SEM images of fractured samples sintered at (a-b) $200{ }^{\circ} \mathrm{C}$ and $(c-d) 300{ }^{\circ} \mathrm{C}$

199

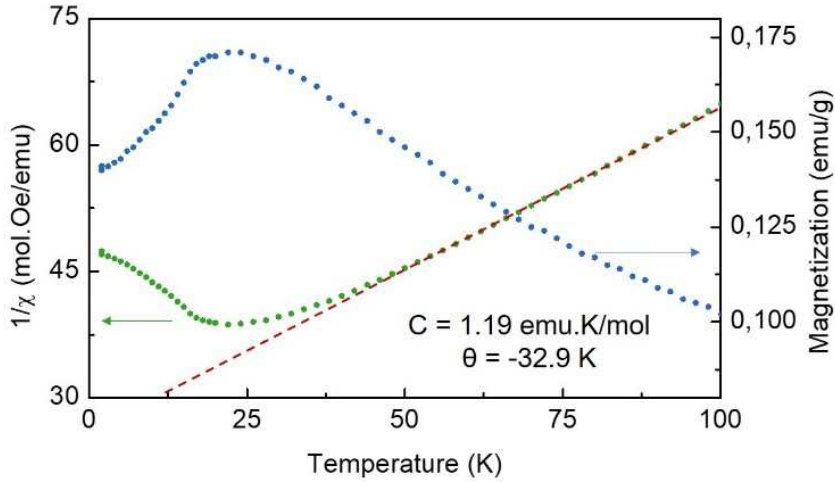

201 Figure 3: Zero field cool measurement on pellet sintered at $300{ }^{\circ} \mathrm{C}-300 \mathrm{MPa}-10 \mathrm{~min}$, green dots correspond to inverse of 202 magnetic susceptibility and blue dots to sample magnetization; red dashed line corresponds to Curie-Weiss fit 


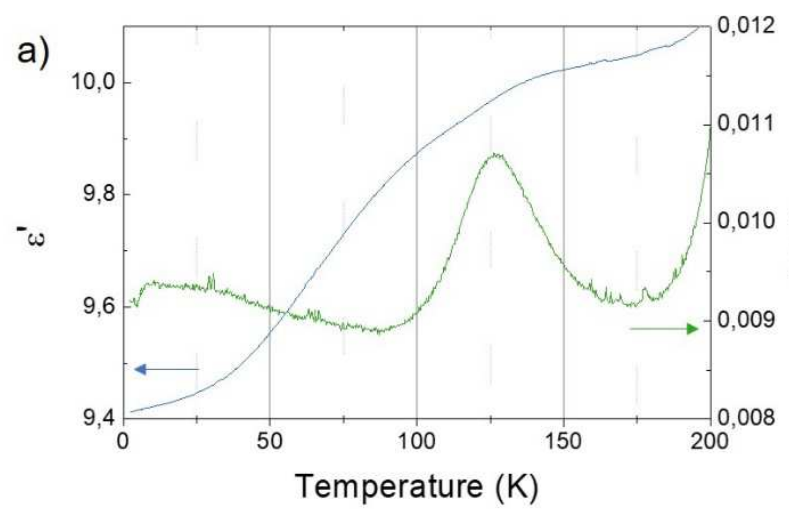

Figure 4: a) Dielectric measurement in the range $2-200 \mathrm{~K}$, measured at $300 \mathrm{kHz}$ min and b) Magnetic field dependence of
permittivity at $300 \mathrm{kHz}$ measured on a pellet sintered at $300{ }^{\circ} \mathrm{C}-300 \mathrm{MPa}-10$

Figure 4: a) Dielectric measurement in the range $2-200 \mathrm{~K}$, measured at $300 \mathrm{kHz}$ min and b) Magnetic field dependence of
permittivity at $300 \mathrm{kHz}$ measured on a pellet sintered at $300{ }^{\circ} \mathrm{C}-300 \mathrm{MPa}-10$

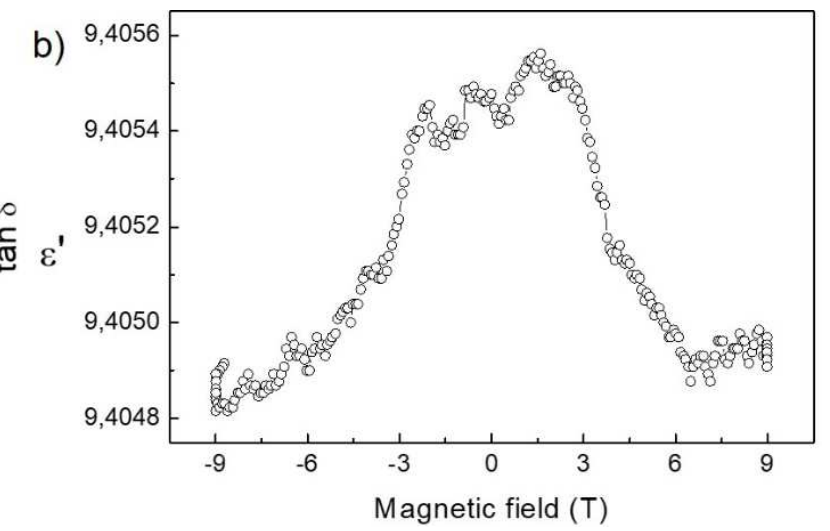

Table 1: Refined lattice parameters for powder and pellets sintered at various temperatures

\begin{tabular}{ccccccc}
\hline Sample & $\begin{array}{c}\text { Sintering } \\
\text { temperature }\left({ }^{\circ} \mathbf{C}\right)\end{array}$ & $\mathbf{a}(\AA)$ & $\mathbf{b}(\AA)$ & $\mathbf{c}(\AA)$ & $\boldsymbol{\beta}\left({ }^{\circ}\right)$ & $\begin{array}{c}\text { Relative } \\
\text { density (\%) }\end{array}$ \\
\hline$\# \mathbf{1}$ & 200 & $7.5175(3)$ & $10.0065(3)$ & $8.2738(3)$ & $105.895(3)$ & $94.1(5)$ \\
$\# 2$ & 250 & $7.5180(2)$ & $10.0055(3)$ & $8.2780(2)$ & $105.901(3)$ & $95.6(5)$ \\
$\# 3$ & 300 & $7.5176(2)$ & $10.0043(2)$ & $8.2729(2)$ & $105.900(3)$ & $98.0(5)$ \\
Powder & - & $7.5197(4)$ & $10.0023(5)$ & $8.2759(5)$ & $105.856(6)$ & - \\
Reference [20] & - & $7.5219(2)$ & $10.0018(3)$ & $8.2729(3)$ & $105.887(3)$ & - \\
\hline
\end{tabular}

Table 2: Comparison of some crystal-chemical and magnetic data for the pyrophosphate series $\mathrm{M}^{I} \mathrm{FeP} \mathrm{O}_{7}\left(\mathrm{M}^{I}=\mathrm{Na}, \mathrm{K}, \mathrm{Cs}, \mathrm{NH}_{4}\right.$, $A g)$.

\begin{tabular}{|c|c|c|c|c|}
\hline Compound & Space group & $\boldsymbol{\theta}(\mathbf{K})$ & $\mathbf{R}_{\mathbf{A}}(\stackrel{\mathrm{A}}{)})$ & Ref. \\
\hline $\mathrm{NaFeP}_{2} \mathrm{O}_{7}$ & $\mathrm{P} 2{ }_{1} / \mathrm{c}$ & -53 & 1,18 & {$[23]$} \\
\hline $\mathrm{AgFeP}_{2} \mathrm{O}_{7}$ & $\mathrm{P} 2_{1} / \mathrm{c}$ & -165.9 & 1,28 & {$[22]$} \\
\hline $\mathrm{KFeP}_{2} \mathrm{O}_{7}$ & $\mathrm{P} 2_{1} / \mathrm{c}$ & -90 & 1,51 & {$[23]$} \\
\hline $\mathrm{NH}_{4} \mathrm{FeP}_{2} \mathrm{O}_{7}$ & $\mathbf{P} 2_{1} / \mathbf{c}$ & -32.9 & 1,55 & This Work \\
\hline $\mathrm{CsFeP}_{2} \mathrm{O}_{7}$ & $\mathrm{P} 2_{1} / \mathrm{c}$ & -64.9 & 1,74 & {$[25]$} \\
\hline
\end{tabular}


214 [1] J.-P. Maria, X. Kang, R.D. Floyd, E.C. Dickey, H. Guo, J. Guo, A. Baker, S. Funihashi, C.A.

215 Randall, Cold sintering: Current status and prospects, J. Mater. Res. 32 (2017) 3205-3218.

216 [2] H. Guo, A. Baker, J. Guo, C.A. Randall, Protocol for Ultralow-Temperature Ceramic Sintering:

217 An Integration of Nanotechnology and the Cold Sintering Process, ACS Nano. 10(11) (2016) 10606-

21810614.

219 [3] S. Funahashi, J. Guo, H. Guo, K. Wang, A.L. Baker, K. Shiratsuyu, C.A. Randall, Demonstration 220 of the Cold Sintering Process Study for the Densification and Grain Growth of ZnO Ceramics, J. Am.

221 Ceram. Soc. 100 (2017) 546-553.

222 [4] S.S. Berbano, J. Guo, H. Guo, M.T. Lanagan, C.A. Randall, Cold sintering process of 223 Li1.5Al0.5Ge1.5(PO4)3 solid electrolyte, J. Am. Ceram. Soc. 100 (2017) 2123-2135.

224 [5] D. Wang, D. Zhou, S. Zhang, Y. Vardaxoglou, W. Whittow, D. Cadman, I. Reaney, Cold225 Sintered Temperature Stable Na0.5Bi0.5MoO4-Li2MoO4 Microwave Composite Ceramics, ACS 226 Sustain. Chem. Eng. 6 (2018) 2438-2444.

227 [6] I.J. Induja, M.T. Sebastian, Microwave dielectric properties of mineral sillimanite obtained by 228 conventional and cold sintering process, J. Eur. Ceram. Soc. 37 (2017) 2143-2147.

229 [7] H. Guo, A. Baker, J. Guo, C.A. Randall, Cold Sintering Process: A Novel Technique for Low230 Temperature Ceramic Processing of Ferroelectrics, J. Am. Ceram. Soc. 99 (2016) 3489-3507.

231 [8] A. Ndayishimiye, S. Buffière, M.A. Dourges, A. Largeteau, M. Prakasam, S. Mornet, O. Kaman, 232 J. Zdenĕk, J. Hejtmánek, G. Goglio, Design of 0-3 type nanocomposites using hydrothermal sintering, 233 Scr. Mater. 148 (2018) 15-19.

234 [9] A. Ndayishimiye, A. Largeteau, M. Prakasam, S. Pechev, M.A. Dourges, G. Goglio, Low 235 temperature hydrothermal sintering process for the quasi-complete densification of nanometric $\alpha$-quartz, 236 Scr. Mater. 145 (2018) 118-121.

237 [10] A. Ndayishimiye, A. Largeteau, S. Mornet, M. Duttine, M.-A. Dourges, D. Denux, M. Verdier, 238 M. Goune, T. Herisson de Beauvoir, C. Elissalde, G. Goglio, Hydrothermal Sintering for Densification of 239 Silica . Evidence for the Role of Water, J. Eur. Ceram. Soc. 38 (2018) 1860-1870.

240 [11] T. Herisson de Beauvoir, F. Molinari, U.-C. Chung-Seu, D. Michau, D. Denux, M. Josse, 241 Densification of MnSO4 ceramics by Cool-SPS: evidences for a complex sintering mechanism and 242 magnetoelectric coupling, J. Eur. Ceram. Soc. 38 (2018) 3867-3874.

243 [12] T. Herisson de Beauvoir, A. Sangregorio, I. Cornu, C. Elissalde, M. Josse, Cool-SPS: an 244 opportunity for low temperature sintering of thermodynamically fragile materials, J. Mater. Chem. C. 6(9) 245 (2018) 2229-2233.

246 [13] D. Sohrabi Baba Heidary, M. Lanagan, C.A. Randall, Contrasting energy efficiency in various 247 ceramic sintering processes, J. Eur. Ceram. Soc. 38 (2018) 1018-1029.

248 [14] X. Zhao, J. Guo, K. Wang, T. Herisson De Beauvoir, B. Li, C.A. Randall, Introducing a ZnO249 PTFE (Polymer) Nanocomposite Varistor via the Cold Sintering Process, Adv. Eng. Mater. (2018) 2501700902. 

sintered V2O5 ceramics and Co-sintered V2O5-PEDOT:PSS composites, J. Eur. Ceram. Soc. 37 (2016) 253 1529-1534.

254 [16] J. Guo, S.S. Berbano, H. Guo, A.L. Baker, M.T. Lanagan, C.A. Randall, Cold Sintering Process 255 of Composites : Bridging the Processing Temperature Gap of Ceramic and Polymer Materials, Adv.

256 Funct. Mater. 26 (2016) 7115-7121.

257 [17] S. Funahashi, H. Guo, J. Guo, A.L. Baker, K. Wang, K. Shiratsuyu, C.A. Randall, Cold sintering 258 and co-firing of a multilayer device with thermoelectric materials, J. Am. Ceram. Soc. 100 (2017) 34882593496.

260 [18] T. Herisson de Beauvoir, V. Villemot, M. Josse, On the first molecular ceramic, obtained by 261 Cool-SPS, submitted.

262 [19] X. Fleury, M.H. Noh, S. Geniès, P.X. Thivel, C. Lefrou, Y. Bultel, Fast-charging of Lithium Iron 263 Phosphate battery with ohmic-drop compensation method: Ageing study, J. Energy Storage. 16 (2018)

264 21-36.

265 [20] D. Grossin, S. Rollin-Martinet, C. Estournès, F. Rossignol, E. Champion, C. Combes, C. Rey, C. 266 Geoffroy, C. Drouet, Biomimetic apatite sintered at very low temperature by spark plasma sintering: 267 Physico-chemistry and microstructure aspects, Acta Biomater. 6 (2010) 577-585.

268 [21] B.F. Alfonso, J.A. Blanco, M.T. Fernández-Díaz, C. Trobajo, S.A. Khainakov, J.R. García, On 269 the crystal structure and thermal decomposition of ammonium-iron(iii) bis(hydrogenphosphate), Dalt. 270 Trans. 39 (2010) 1791-1796.

271 [22] K. V. Terebilenko, A.A. Kirichok, V.N. Baumer, M. Sereduk, N.S. Slobodyanik, P. Gütlich, 272 Structure and magnetic properties of AgFeP2O7, J. Solid State Chem. 183 (2010) 1473-1476.

273 [23] D. Riou, N. Nguyen, R. Benloucif, B. Raveau, LiFeP2O7 : Structure and magnetic properties, 274 Mater. Res. Bull. 25 (1990) 1363-1369.

275 [24] T. Moya-Pizarro, R. Salmon, L. Fournes, G. Le Flem, B. Wanklyn, P. Hagenmuller, Etudes 276 cristallographique magnétique et par résonance Mössbauer de la variété de haute température du 277 pyrophosphate NaFeP2O7, J. Solid State Chem. 53 (1984) 387-397.

278 [25] E. Dvoncova, K.-H. Lii, Synthesis, crystal structure, and magnetic susceptibilities of CsFeP2O7 279 and RbFeP2O7, J. Solid State Chem. 105 (1993) 279-286.

280 [26] S. Gentil, D. Andreica, M. Lujan, J. Rivera, F. Kubel, H. Schmid, Synthesis, structure and 281 magnetic susceptibility of $\mathrm{KCrP} 2 \mathrm{O} 7$, a potential antiferromagnetic magnetoelectric, Ferroelectrics. 204 282 (1997) 35-44. 